\title{
COLLABORATION TO IMPROVE CROSS-RACE FACE RECOGNITION: WISDOM OF THE MULTI-RACIAL CROWD?
}

\author{
Jacqueline G. Cavazos \\ U. of Texas at Dallas
}

\author{
Geraldine Jeckeln \\ U. of Texas at Dallas
}

\author{
Alice J. O'Toole \\ U. of Texas at Dallas
}

September 13, 2021

\begin{abstract}
Collaborative "wisdom-of-crowds" decision making improves face identification accuracy over individuals working alone. We examined whether collaboration improves both own- and other-race face identification. In Experiment 1, participants completed an online face-identification task on their own and with a same-race partner (East Asian dyads, $\mathrm{N}=27$; Caucasian dyad, $\mathrm{N}=31$ ). Collaborative decisions were completed as part of a social dyad (completing the task together) and a non-social dyad (individual scores fused independently). Social and non-social collaboration improved ownand other-race face identification accuracy equally. In Experiment 2, we examined the impact of racial diversity on collaboration for different-race dyads $(\mathrm{N}=25)$, East Asian same-race dyads $(\mathrm{N}=$ $25)$, and Caucasian same-race dyads $(\mathrm{N}=28)$. Performance improved equivalently for same- and different-race dyads. Collaboration can be a valuable tool for improving own- and other-race face identification in social and non-social settings.
\end{abstract}

\section{Introduction}

People recognize faces of their own race more accurately than faces of "other" races [1]. This "other-race-effect" (ORE) occurs in laboratory [2] and real-world settings (e.g., eyewitness identification) [3]. Potential strategies for improving other-race face recognition include individuated training [4, 5, 6, 7, 8], increased developmental exposure [9, 10, 11], caricatured exemplars [12], increased learning time [13], and learning identities from multiple images [14]. However, there are limitations to each of these strategies. For example, individuated training is time consuming [6, 5, 8] and learning identities from caricatured exemplars or multiple images is impractical in applied scenarios (e.g., eyewitness identification). Increased developmental exposure cannot be leveraged to help adults. Consequently, there remains a need to explore ways to improve other-race face recognition.

One promising strategy is to exploit collaborative "wisdom-of-crowds" decision making [15]. Face identification for own-race faces is more accurate when decisions are made by pairs/groups of people than when they are made individually [16]. Collaboration improves face-identification accuracy in both social [17, [18] and non-social scenarios [19, 20, 18]. In social collaboration, two or more individuals work together to produce a single response. In non-social collaboration, individual item responses are averaged to obtain a group judgment without social interaction (i.e., blind fusion). Social and non-social collaboration yield equivalent benefits for face recognition [18]. Whether collaborative decision-making improves accuracy for other-race face identification, has not been studied.

Social and non-social collaboration operate via distinct mechanisms. In social collaboration, performance is driven by the higher-performing individual [17, 18]. Because social dyads rely on the more accurate member of a pair [18], low-performing individuals benefit more [17]. Jeckeln et al. (2018) used computational models [21] to demonstrate that dyads depend on social feedback to determine the more accurate member of the dyad (cf. Behavior-Feedback model, [21] [[21]]). Notably, social collaboration considers overall individual accuracy when grouping individuals. In contrast, non-social collaboration combines individual responses at the item-level and considers each dyad member and each stimulus item equally [19, 20, 22] and therefore exploits strengths of both individuals. Blind fusion works best when individual strategies are both different and effective [23, 22]. For example, fusing algorithms with different 
computational strategies [23] as well as fusing algorithms and humans (with seemingly different strategies) yields performance improvements [22].

Although the effect of diversity (including racial diversity) in group decision making has been considered previously, to date, no study has examined the role of racial diversity in face identification accuracy. We explore the question of how the racial diversity of the individuals in a dyad affects both social and non-social collaboration on a face-identification task. We might expect that the combination of two different race individuals with strategies of recognition tuned, each for faces of their own race, would yield strong benefits, and greater overall performance for different-race dyads. Alternatively, assuming that people have higher ability for own- vs other-race face recognition, introduces yet another dimension when considering specifically same-race face identification accuracy. Diversity and ability often compete with one another [24, 25, 26]. High ability groups tend to be inherently less diverse, and highly diverse groups tend to have a greater range of individual ability. From the social perspective, racial diversity in groups increases creativity and thoughtful responses [27, 28], but also conflict and distrust, compared to homogeneous groups [24, 29]. Combined, the literature suggests that the effect of race on collaboration may be complex.

In Experiment 1, we evaluated the benefits of collaboration for own- and other-race face identification accuracy. We hypothesized that collaboration would benefit performance in social and non-social situations for own- and other-race face identification. In Experiment 2, we compared racially diverse (East Asian and Caucasian) dyads vs racially homogeneous dyads (two East Asians, two Caucasians). If ability is a stronger factor than diversity, performance will be better in homogeneous dyads. If strategy diversity is a stronger factor than ability, performance will be better in racially diverse dyads.

\section{Experiment 1}

In Experiment 1, we addressed two main questions. First we examined if collaboration (both social and non-social) improves both own- and other-race face identification accuracy. Second, we tested the effectiveness social and non-social collaboration for improving face-identification accuracy. Social and non-social collaboration have been shown to yield similar face identification accuracy improvements in previous work [18]. However, the complex effects of race on face identification suggests these two collaborative strategies might impact own- and other-race face identification differently.

\section{Methods}

\subsection{Participants}

Participants $(N=116)$ were recruited in same-race dyads (East Asian dyads; $\mathrm{N}=27$ )/ Caucasian dyads; $\mathrm{N}=31$ ) from the University of Texas at Dallas (UTD) online research participant pool, SONA. One research exposure class credit was given as compensation for participation. A power analysis using PANGEA (v0.2) [30] revealed that a total of 108 participants (54 dyads) was needed in order to detect a medium effect size $(\mathrm{d}=0.5)$ with a power of .80 . This power was selected to detect the full 2 (within-subjects: stimulus race) x 3 (within-subjects: group) x 2 (between-subjects: participant race) interaction. Eligible participants self-identified as either East Asian or Caucasian (but not both), had normal or corrected-to-normal vision, and were at least 18 years old. Race eligibility was determined based on self-report via an eligibility survey using Qualtrics ([31]). Three Caucasian participants' (and their partners') data were removed because once they completed the experiment, they reported that they did not identify as Caucasian. Three participants, (two Caucasian and one East Asian) and their partner's data were removed due to low performance (less than 2 SD below the mean). The final analysis was computed on 104 participants or 52 dyads (26 East Asian dyads, and 26 Caucasian dyads).

\subsection{Stimuli}

Face images (40 East Asian, 40 Caucasian) were selected from the Notre Dame dataset [32] (See Figure 11). A state-of-the-art face recognition algorithm based on Deep Convolutional Neural Networks, A2017b [33], was used to screen for challenging image stimuli. This algorithm has similar identification accuracy for both East Asian and Caucasian faces. Challenging image pairs were selected by choosing same-identity pairs with the lowest similarity scores, and different-identity pairs with the highest similarity scores. For the final 80 image pairs, the A2017b algorithm

\footnotetext{
${ }^{1}$ Although initially designed as an in-person study, the events surrounding the 2019 Coronavirus pandemic did not permit us to carry out this experiment as planned. Therefore, all experimental procedures for Experiments 1 and 2 were conducted online to comply with social-distancing requirements.
} 

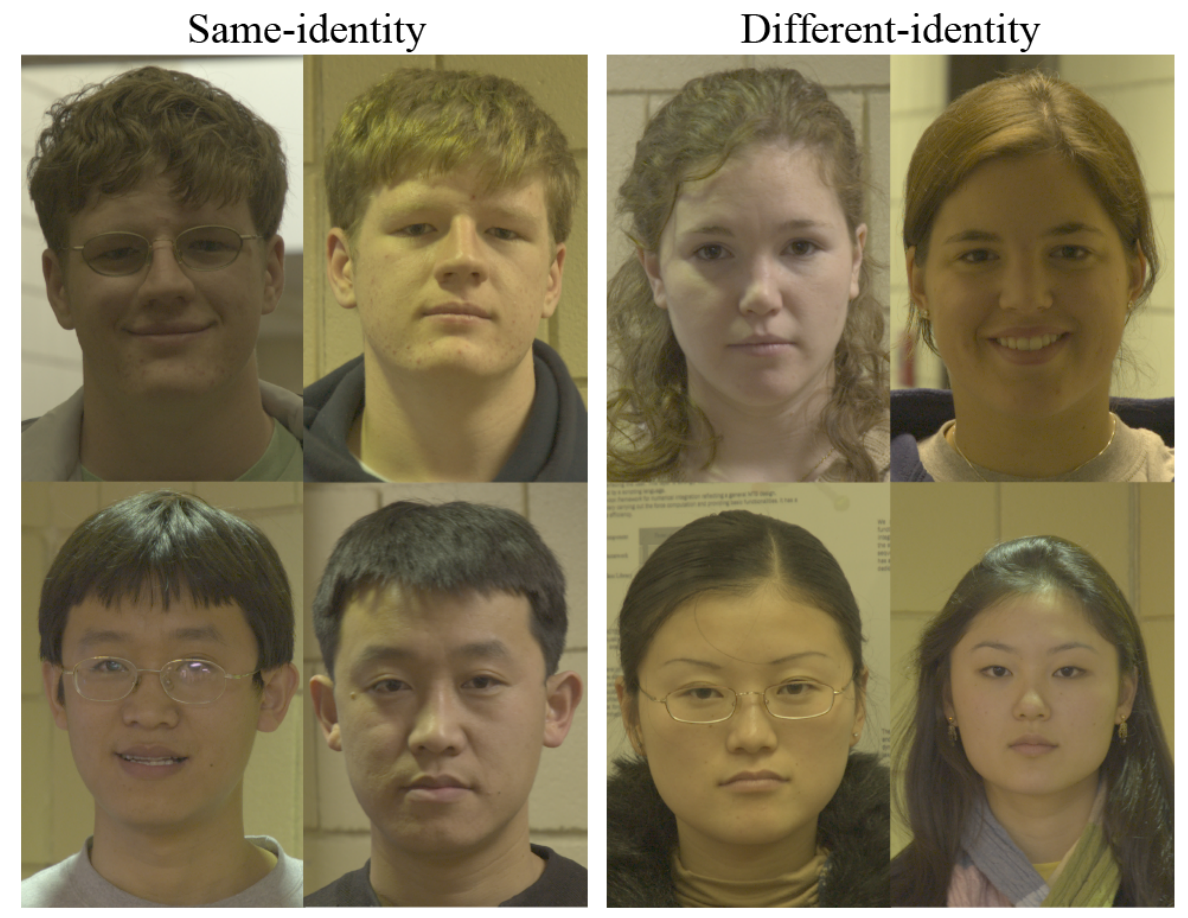

Figure 1: Exp. 1 and 2: stimuli of Caucasian (top panel) and East Asian (bottom panel) same-identity pairs (left panel), and different-identity pairs (right panel).

had comparable accuracy for East Asian $(\mathrm{AUC}=.403)$ and Caucasian faces $(\mathrm{AUC}=.403)$. The image pairs were divided into two sets: Set A (20 East Asian/20 Caucasian) and Set B (20 East Asian/20 Caucasian). The two image sets were used to counterbalance the images across conditions (individual and social-dyad). All images were cropped to reveal the face and neck area only.

\section{Procedure}

Participants were recruited in same-race dyads (see Experiment 2 for different-race pairs). After signing up, participants received a Webex Meetings link to join the study virtually. The experimental session began once both participants joined the Webex meeting (experiment). Participants were encouraged to turn on their webcam and were required to have a working microphone/speaker. After completing the consent process, participants received verbal instructions for the experimental task. All experimental tasks were completed virtually through the use of the "screen share" and the "screen control features" on Webex. These functions enabled participants to view the researchers' computer screen and use their own mouse and keyboard to navigate the experiment. Participants saw two faces (image pairs) on the screen side-by-side at a time, and were then asked if the images pairs represented the same person, or two different people. Responses were made using a 5-point Likert scale $(+2$ : Sure they are the same person, +1 : Think they are the same person, 0: Do not know, -1: Think they are different people, -2: Sure they are different people). Participant responses were recorded by clicking on the rating scale on the computer screen. This task was completed twice, once individually (individual), and once with a partner (social dyad). Task order was counterbalanced, such that half of the participants completed the individual task and then the social-collaboration task, and half completed the social-collaboration task first and then the individual task.

Set A and Set B were counterbalanced also, such that half the participants completed the individual task on Set A (and the partner task with Set B) and half completed the individual task with Set B (and the partner task with Set A). The task was self-paced and all responses were recorded using mouse clicks on the screen. After completing both tasks, a demographic and social dyad experience questionnaire was administered. 


\subsection{Individual}

While one member of the pair completed the individual task, their study partner was transferred to the Webex Meeting "lobby". While in the "lobby", the study partner could not hear or see any of the research activities, but they were not removed from the Webex call. There was no social interaction between participants at this time.

\subsection{Social Collaboration}

During the social-collaboration condition, both participants were invited back into the Webex meeting where they were able to see the screen and virtually interact with each other. Since the Webex screen control feature can only be granted to one user at a time, "clicker" duties were randomly assigned to the one participant, before the experiment began. Participants were instructed to work together to come up with a single decision for each image pair. There were no instructions on how participants should select the final answer or how rating disagreements should be resolved.

\subsection{Non-Social Collaboration}

Item-by-item responses completed in the individual condition were fused, or averaged, together for each dyad. This fusion created a composite fused score for each dyad on every item. This new fused score was used to compute performance on the non-social collaboration condition.

\subsection{Social Dyad Experience Questionnaire}

To measure participants' experience completing the task with a partner and virtually, a ten-question survey was administered after the demographic survey (see Appendix A). This included five questions related to the participant's experience working with their partner (e.g., 'My partner and I worked well together', 'I felt comfortable voicing my opinion to my partner') rated from Strongly Agree to Strongly Disagree. Participants were asked also to rate their contribution and their partner's contribution on the task (a 50\%-50\% response denoted equal contribution). Furthermore, participants were also asked to report which facial features (eyes, nose, mouth, other, etc.) they focused on when making responses on their own, and with their partner. Given the virtual nature of this study, three additional questions were asked to gauge participants experience completing the task virtually (e.g., 'Taking the experiment online made it difficult to complete the task.', 'Connectivity or internet issues made it difficult to complete the task.', 'Overall, my experience completing the task online was enjoyable').

\section{Analysis and Results}

The data were submitted to a 2 (stimulus race: East Asian/Caucasian) x 2 (participant race: East Asian/Caucasian) x 3 (group: Individual/Non-Social Dyad/Social Dyad) mixed-ANOVA design with stimulus race and groups as withinsubjects factors and participant race as a between-subjects factor. The dependent variable (performance) was measured as the area under the receiver operating characteristic curve (AUC).

\subsection{Race Effects}

Results showed no effect of participant race. However, there was an effect of stimulus race $(F(1,102)=7.84, M S e=$ $\left..008, p=.006, \eta_{p}^{2}=.07\right)$. Face identification accuracy was greater for East Asian faces $(M=.76, S D=.11)$ than for Caucasian faces $(M=.74, S D=.10)$. This stimulus effect was qualified by the presence of a significant interaction between stimulus and participant race (i.e., a partial other-race effect $)\left(F(1,102)=5.03, M S e=.008, p=.03, \eta_{p}^{2}=.05\right)$. East Asian participants had a greater accuracy for East Asian faces $(M=.77, S D=.11)$ compared to Caucasian faces $(M=.73, S D=.11)$. In contrast, Caucasian participants had comparable accuracy for Caucasian faces $(M=.76, S D=$ $.10)$ and East Asian faces $(M=.76, S D=.10)$ (see Figure 2).

\subsection{Collaboration benefits}

To test the benefits of collaboration, overall accuracy was measured across three conditions: individuals, non-social dyads, and social-dyads. As expected, there was an effect of group condition $(F(2,204)=2.71, M S e=.008, p<.0001$, $\left.\eta_{p}^{2}=.21\right)$ (see Figure 3). Planned comparisons revealed that non-social dyads $(M=.78, S D=.11)$ had greater accuracy than individuals $(M=.72, S D=.11)(t(204)=7.12, p<.0001)$. Similarly, social-dyads $(M=.76, S D=.09)$ had a greater accuracy than individuals $(t(204)=5.19, p<.0001)$. There were no differences between social and non-social dyads $(t(204)=1.92, p=.1675)$. No other collaboration interactions were significant, which indicates comparable collaboration benefits for own- and other-race faces (see Figure 4). 


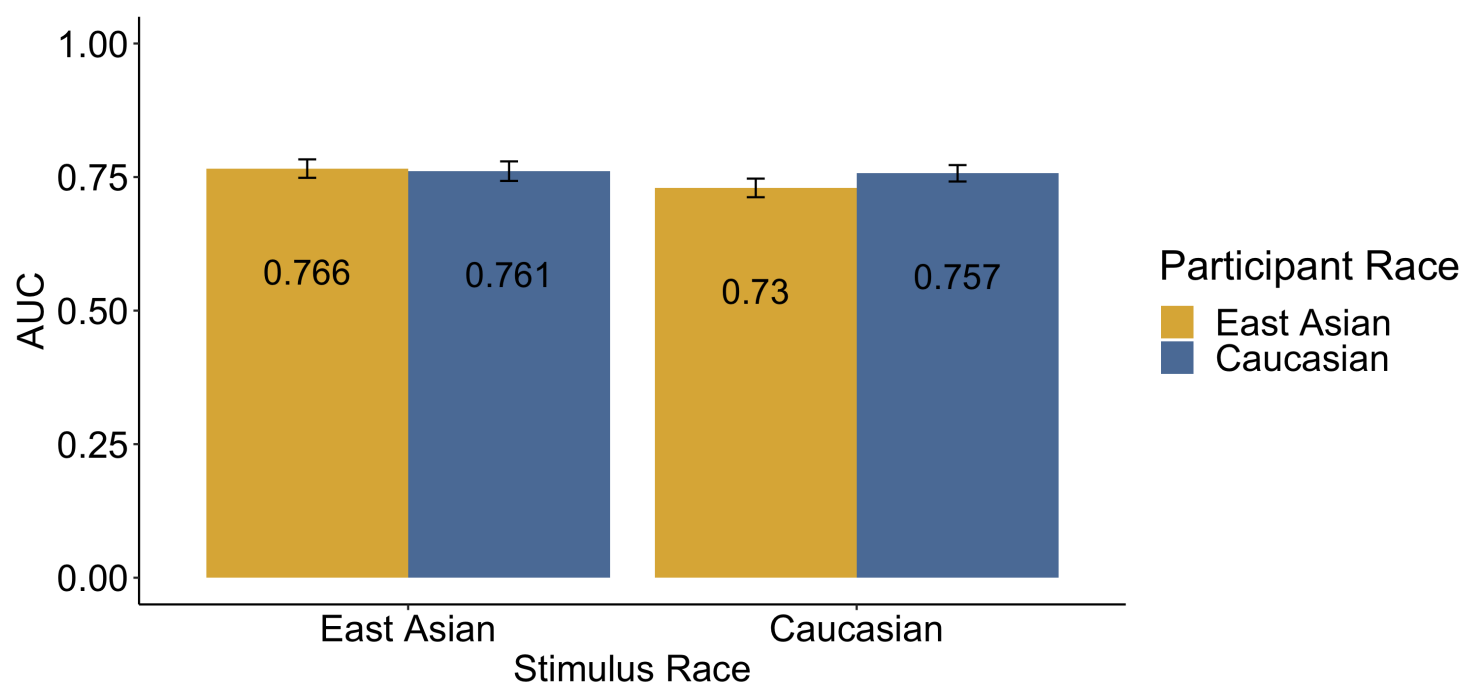

Figure 2: Face-identification accuracy for own- and other-race faces. Accuracy (AUC) results for East Asian participants (yellow) and Caucasian participants (blue). East Asian participants had a greater identification accuracy for East Asian faces compared to Caucasian faces. Caucasian participants had comparable accuracy for Caucasian faces and East Asian faces.

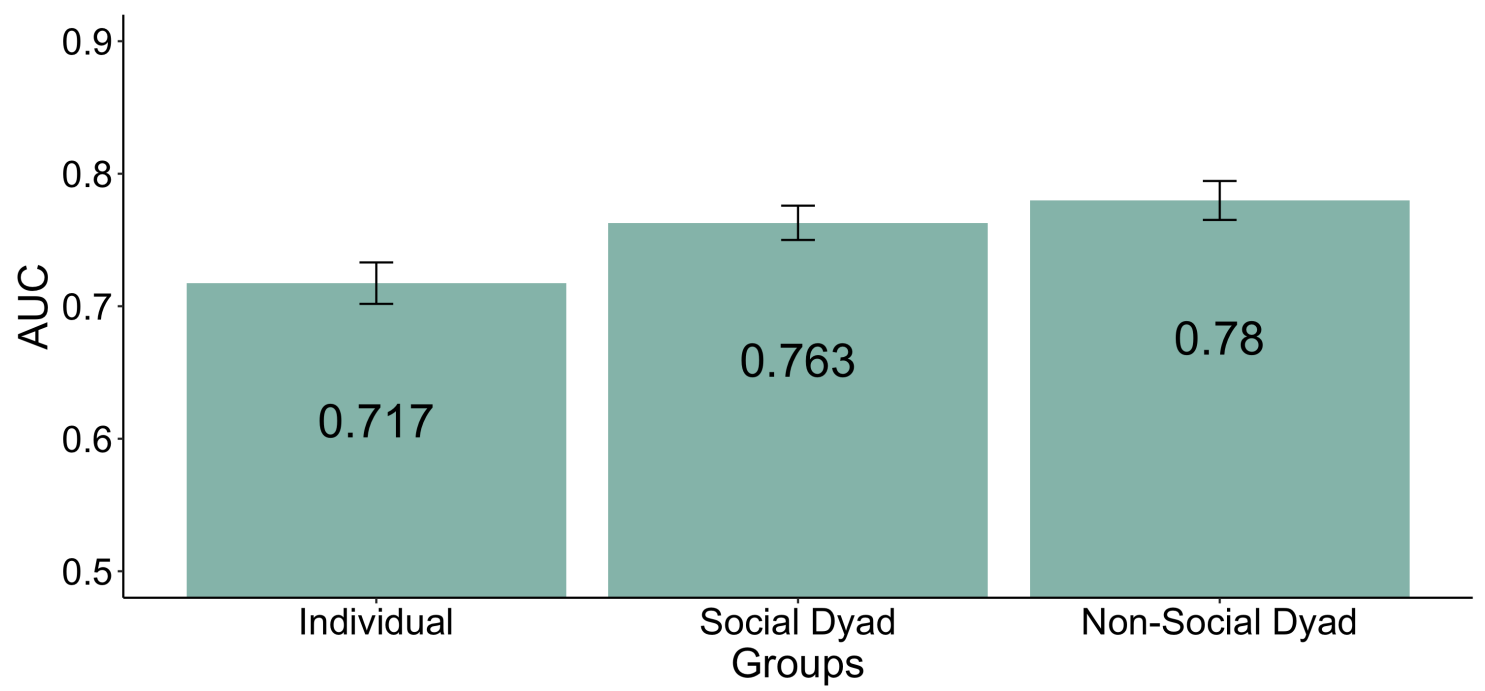

Figure 3: Face-identification accuracy (AUC) for Individuals, Social dyads, and Non-Social Dyads. Social and nonsocial dyad accuracy was greater than individual performance. There were no performance differences between social and non-social collaboration. 


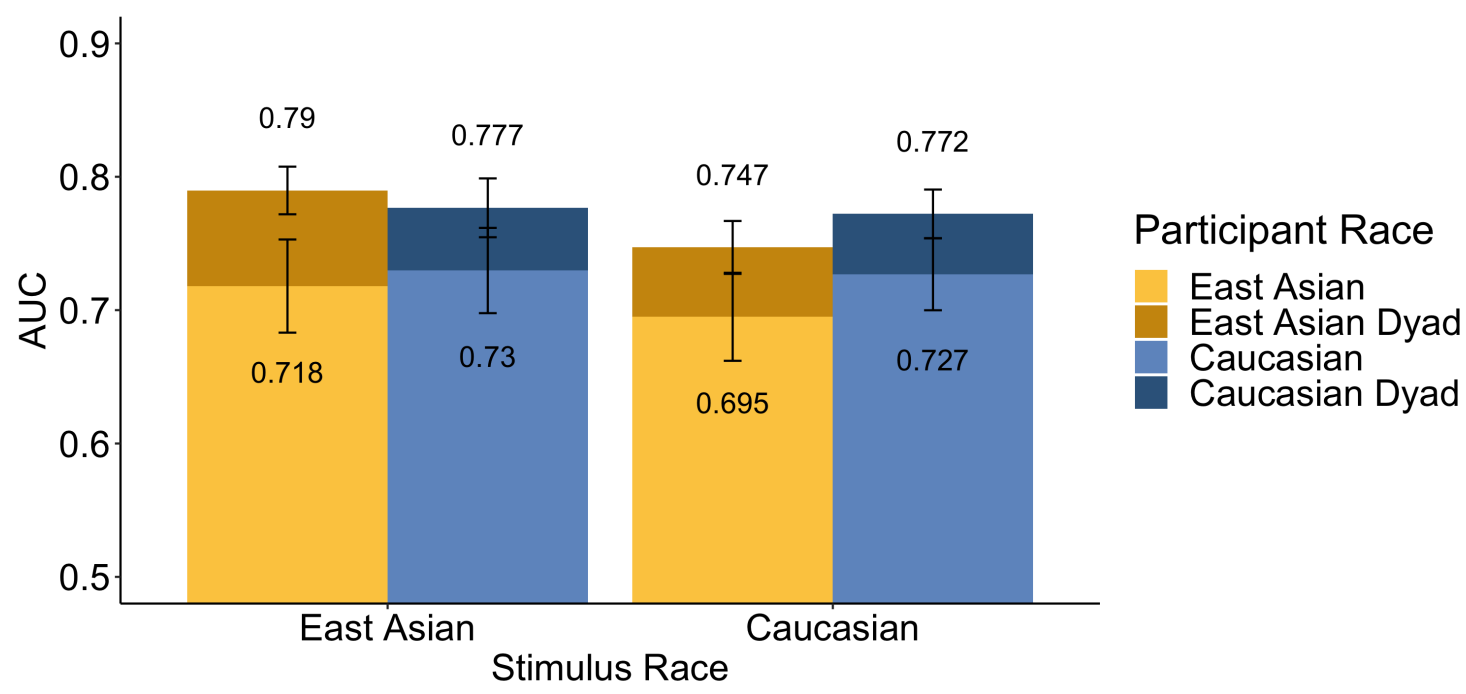

Figure 4: Collaboration benefits for own- and other-race faces collapsed across collaboration type (social and non-social collaboration). Face-identification accuracy for East Asian individuals (yellow), East Asian dyads (dark yellow), Caucasian individuals (blue) and Caucasian dyads (dark blue) on own- and other race-faces. Collaboration improved accuracy for both East Asian and Caucasian individuals for both race face stimulus.

\subsection{Social experience survey}

Generally, participants reported that they worked well with their partner. The majority (87-94\%) of participants agreed with the following statements: "My partner and I worked well together." (37\%: Agree; 57\%: Strongly agree), "I would gladly work with my partner again on other tasks." (33\%: Agree; 54\%: Strongly agree), and "I felt comfortable voicing my opinion to my partner." (30\%: Agree; 61\%: Strongly agree). On average, participants reported that their partner and themselves contributed equally to the task (self: 50\%, partner: 40\%). Notably, although participants worked well with their partner, about a third of participants strongly disagreed with the statement "I felt comfortable letting my partner know when I disagreed." (31\%: Agree; 30\%: Strongly disagree). For questions related specifically to completing the task online, most participants disagreed with the following statements: (e.g., 'Taking the experiment online made it difficult to complete the task.'(36\%: Disagree; 43\%: Strongly disagree), 'Connectivity or internet issues made it difficult to complete the task.'(30\%: Disagree; 53\%: Strongly disagree) and agreed with 'Overall, my experience completing the task online was enjoyable')(42\%: Agree; 52\%: Strongly Agree).

\section{Experiment 1: Discussion}

Collaboration improves identification accuracy for both own- and other race faces. Thus, it is a robust strategy for increasing face-identification accuracy, both within and across race. Social and non-social modes of collaboration proved equally beneficial, consistent with [18]. Social interaction between individuals is not a prerequisite for collaborativedecision making improvements, for either own- and other-race individuals. Participants' generally positive interactions with their same-race dyad partner in the social dyad task suggests limited conflict during the task. However, about a third of participants did not feel comfortable telling their partner when they disagreed. Participants' hesitation to voice their opinions may have been due to several factors associated with working in dyads (e.g., personality differences, feeling intimidated, put off by their partner).

\section{Experiment 2}

Next, we explored how group racial diversity can impact collaboration benefits. Group diversity in decision-making tasks can produce both positive and negative outcomes [34, 35]. Diversity promotes innovative and more thoughtful solutions/responses [27, 24], but it can also heighten distrust and conflict [24, 29]. 
We also examined the impact of other-race experience via an individuated experience survey. Other-race experience is a commonly explored explanation for the ORE, but one that has yield mixed results. Increased other-race experience can mitigate the ORE [1, 36, 37, 38] or have no influence at all [1, 39, 40]. We note at the outset however, that the survey used here was not designed to capture other-race experiences in the context of social-distancing which was in effect when the present data were collected. The misalignment between the survey's original scope and the unique socially-distanced interactions in effect during this study, made it difficult to draw meaningful conclusions obtained from the other-race experience survey. Survey details can be found in Supplemental Materials and we will return to the potential effects of socially-distanced interactions in the discussion.

\section{Methods}

\subsection{Participants}

For Experiment 2, participant recruitment, materials, and restrictions were the same as in Experiment 1, except for the demographic composition of the dyads. For Experiment 2, recruitment included both same-race and different-race dyads. Participants were recruited in same-race pairs (East Asian/East Asian and Caucasian/Caucasian) as well as different-race pairs (East Asian/Caucasian). A power analysis using PANGEA (v0.2) [30] indicated that a total of 162 participants (81 dyads) was needed in order to detect a medium effect size $(\mathrm{d}=0.5)$ with a power of .80 . This power was selected to detect the full 2 (within-subjects: stimulus race) x 3 (within-subjects: group) x 3 (between-subjects: dyad race) interaction. Eligibility requirements were the same as in Experiment 1: a) self-identify as either East Asian or Caucasian (but not both), b) have normal or corrected-to-normal vision, and c) be at least 18 years old. Race eligibility was determined based on self-report via an eligibility survey using Qualtrics ([31]). Two East Asian participants' (and their partners') data were removed, because they reported that they did not identify as East Asian after they completed the experiment. The final analysis was computed on 154 participants or 77 dyads (24 East Asian Dyads, 25 East Asian/Caucasian Dyads and 28 Caucasian Dyads).

\subsection{Stimuli and Materials}

The stimuli used in Experiment 2 were identical to those used in Experiment 1.

\section{Procedure}

The procedure for Experiment 2 was identical to that used in Experiment 1 with the addition of the individual other-race experience survey (see Supplemental). Participants completed both individual and social dyad face identifications (counterbalanced), the demographic and social dyad experience survey, and an individuation experience survey. During the Webex meeting, as one participant completed the individual face identification task, their study partner completed the individuation survey. Once again, the non-social dyad condition was created "synthetically" after data collection was completed.

\section{Analysis and Results}

The data were submitted to a 2 (stimulus race: East Asian/Caucasian) x 3 (dyad race groups: East Asian/East Asian, Caucasian/Caucasian, East Asian/Caucasian) x 3 (group: individual/non-social dyad/ social dyad) mixed-ANOVA design with stimulus race and groups as within-subject factors and dyad race groups a between-subject factor. Faceidentification accuracy (dependent variable) was once again measured by AUC.

\subsection{Race Effects}

There was no effect of dyad composition, such that accuracy for different-race dyads (East Asian/Caucasian dyads; $M=.78, S D=.11$ ) was comparable to both types of same-race dyads (East Asian/East Asian; $M=.77, S D=.11$ and Caucasian/Caucasian dyads; $M=.77, S D=.11$ ) (see Figure 5). As with Experiment 1, there was an effect of stimulus race $\left(F(1,151)=6.06, M S e=.01, p<.0001, \eta_{p}^{2}=.29\right)$. Face-identification accuracy was greater for East Asian faces $(M$ $=.80, S D=.11)$ than for Caucasian faces $(M=.75, S D=.11)$. This stimulus effect was qualified again by the presence of a significant interaction between stimulus race and dyad composition $\left(F(2,151)=3.56, M S e=.010, p<.03, \eta_{p}^{2}=.05\right)$. (see Figure 6). Again a partial ORE as we observed in Exp 1 was mostly likely caused by East Asian dyad's difficulty with Caucasian face images. The combination of two East Asian individuals working together to identify Caucasian faces resulted in the lowest performance accuracy. Performance for East Asian faces was equivalent across the three different racial dyad compositions. No other interactions were significant. 


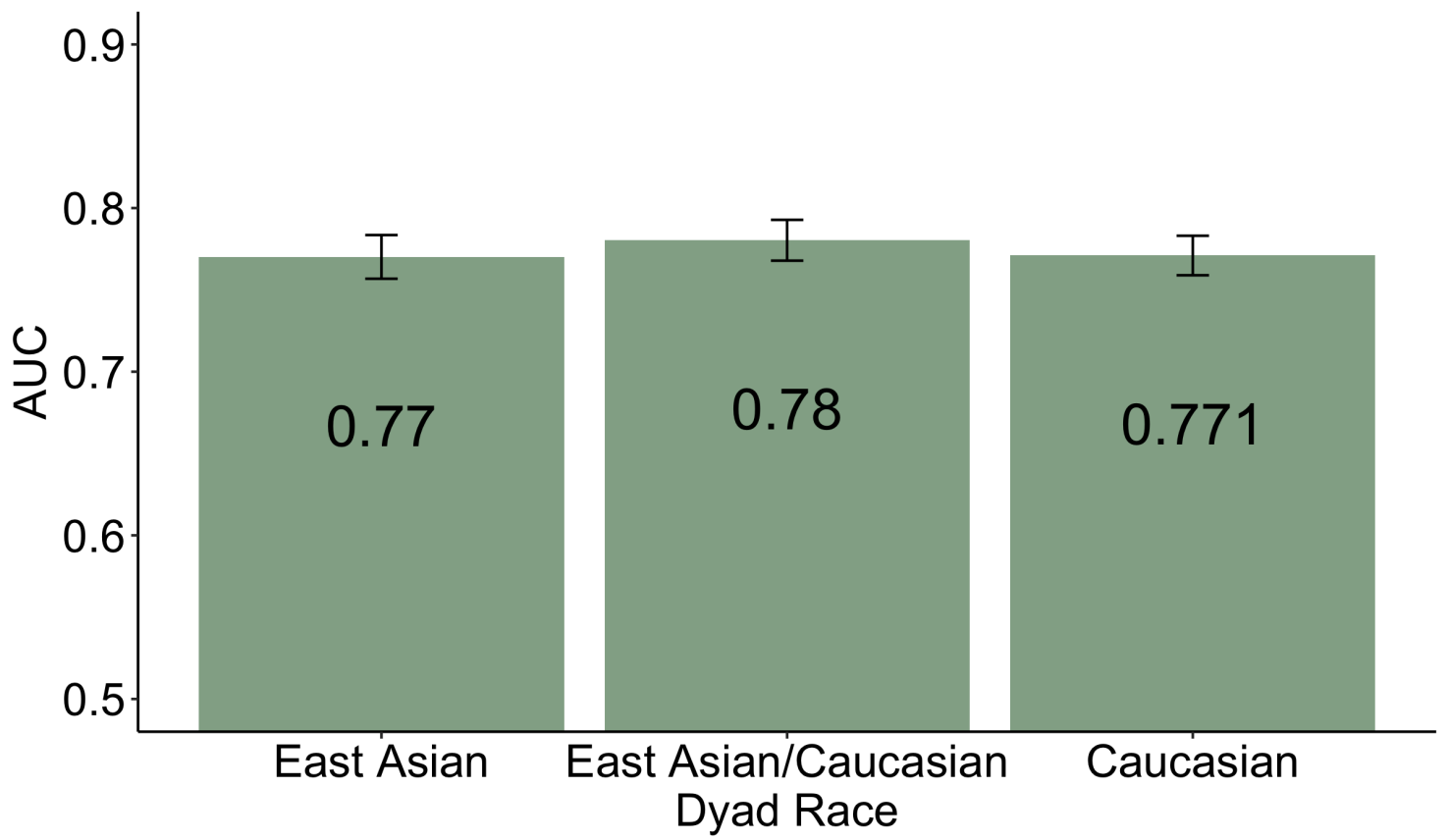

Figure 5: Overall accuracy for different-race (East Asian/Caucasian) and same-race dyads (East Asian/East Asian and Caucasian/Caucasian) was comparable.

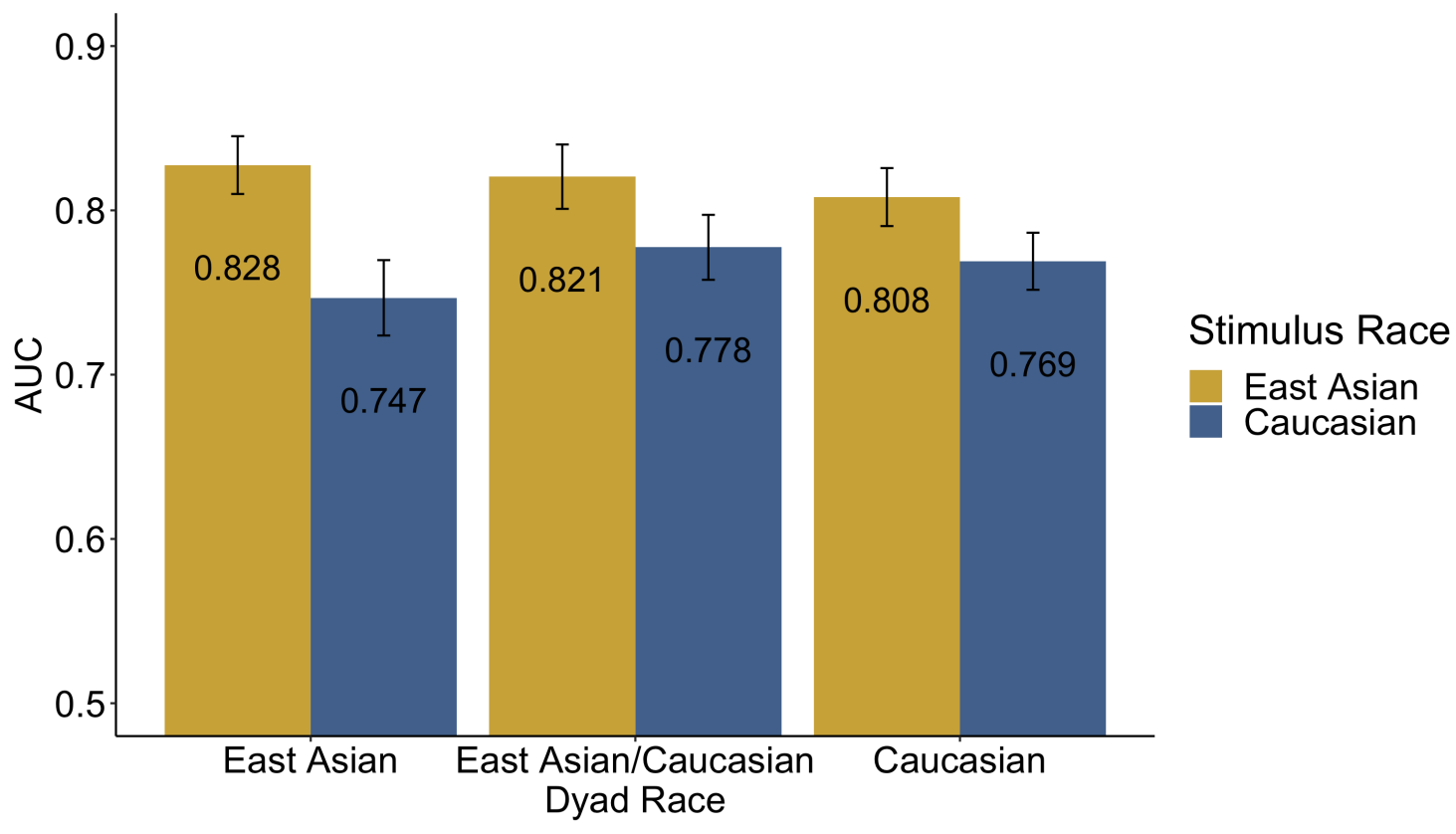

Figure 6: Accuracy for different-race (East Asian/Caucasian) and same-race dyads (East Asian/East Asian and Caucasian/Caucasian) on Caucasian (blue) and East Asian (yellow) faces. This shows that differences across dyad race depends on the stimulus race. East Asian same-race dyads have greater accuracy for same-race faces, but this is not true for Caucasian same-race dyads. For Caucasian dyads accuracy is also greater for East Asian faces. 


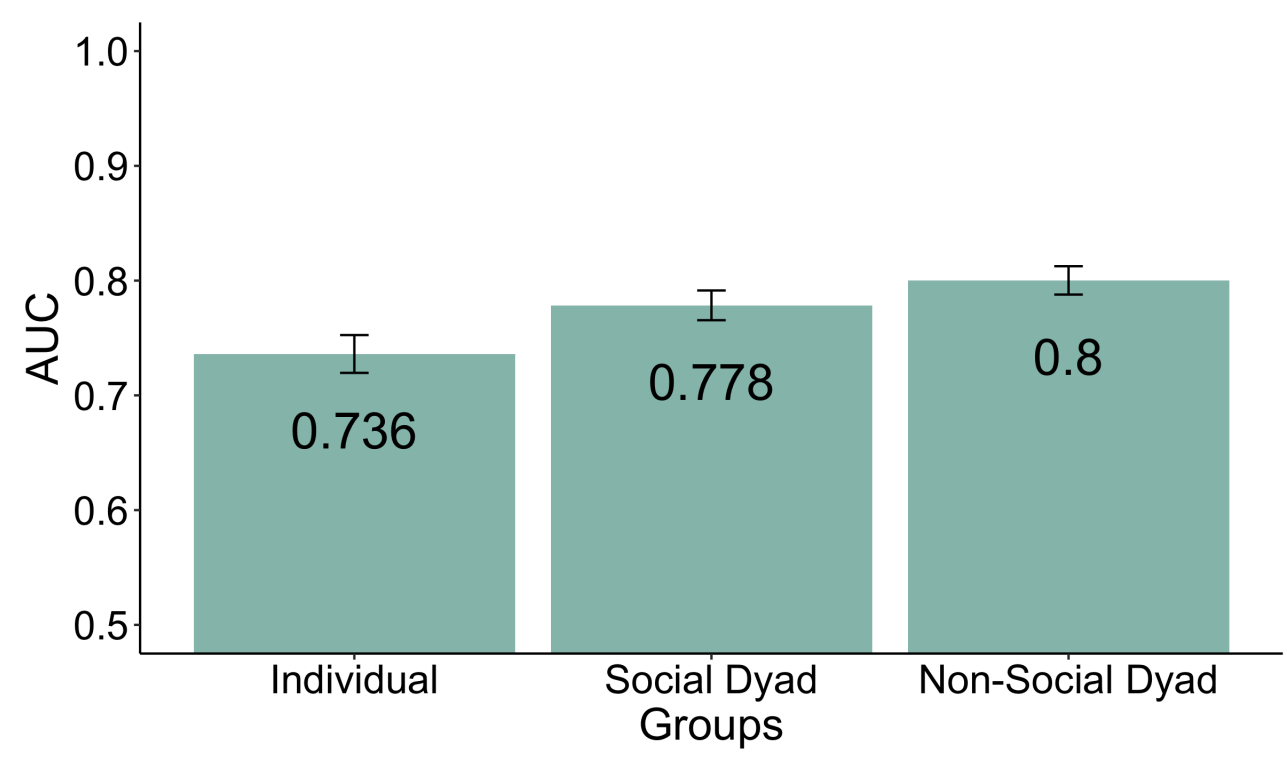

Figure 7: Collaboration benefits for overall accuracy. Both non-social dyads and social dyads had greater identification accuracy than individuals. In addition, non-social dyads had greater identification accuracy than social dyads.

\subsection{Collaboration benefits}

As expected, there was an effect of collaboration type $\left(F(2,302)=3.45, M S e=.010, p<.0001, \eta_{p}^{2}=.19\right)($ see Figure 6). Pair-wise comparisons showed that once again, social dyads $(M=.78, S D=.08)$ and non-social dyads $M=.80, S D=$ $.08)$ were more accurate than individuals $(M=.74, S D=.10)(p<.0001)$. Surprisingly, and in contrast to Experiment 1 , there was a difference between social dyads and non-social dyads, such that overall accuracy was greater for non-social dyads compared to social dyads $(p=.01)$ (see Figure 7). Given that this result was inconsistent with Experiment 1, and given that the primary difference between Experiment 1 and Experiment 2 is the inclusion of different-race dyads, one additional exploratory analysis was conducted. An analysis of social versus non-social collaboration without the different-race dyads revealed that the advantage of the non-social dyad had disappeared. These results replicate Experiment 1 and suggest that the benefit of social collaboration may have been due to the different-race dyads. For completeness, and to explore this post-hoc analysis finding, the dyad composition means as a function of collaboration type are as follows: East Asian Social Dyads $(M=.77, S D=.13)$ and Non-Social Dyads $(M=.81, S D=.11)$; East Asian/Caucasian Social Dyads $(M=.79, S D=.11)$ and Non-Social Dyads $(M=.81, S D=.10)$; Caucasian Social Dyads $(M=.78, S D=.09)$ and Non-Social Dyads $(M=.79, S D=.10)$.

\subsection{Social experience survey}

Generally, participants seemed to work well with their partner. The majority of participants agreed with the following statements: "My partner and I worked well together." (51\%: Strongly agree; 41\%: Agree), "I would gladly work with my partner again on other tasks." (52\%: Strongly agree; 35\%: Agree), and "I felt comfortable voicing my opinion to my partner." (55\%: Strongly agree; 34\%: Agree). Furthermore, on average, participants reported that both themselves and their partner contributed equally to the task. Notably, participants worked well with their partner and a majority of participants agreed with the statement "I felt comfortable letting my partner know when I disagreed."(21\%: Strongly disagree; 43\%: Agree). For questions related specifically to completing the task online, most participants disagreed with the following statements: (e.g., 'Taking the experiment online made it difficult to complete the task.'(36\%: Disagree; 38\%: Strongly disagree), 'Connectivity or internet issues made it difficult to complete the task.'(23\%: Disagree; 55\%: Strongly disagree) and agreed with 'Overall, my experience completing the task online was enjoyable')(40\%: Agree; 48\%: Strongly Agree). 


\section{Experiment 2: Discussion}

At the level of overall performance, completing the task in pairs increased overall face identification performance, regardless of dyad composition. Combined with the lack of an interaction between dyad composition and collaboration type (social and non-social dyads) this supports the claim that combining the strategies of two individuals increases accuracy for both own- and other-race faces. At a more detailed level of analysis, the interaction we observed between dyad composition and stimulus race was driven mostly by differences in identification accuracy for Caucasian faces. Across all dyad compositions, accuracy for East Asian faces was consistent.

Both collaboration types resulted in greater face identification accuracy compared to individual performance. Seemingly inconsistent with Experiment 1, and an unexpected result here was performance differences in collaboration type. Non-social collaboration accuracy was greater than social collaboration. Experiment 2 differed from Experiment 1 because it included different-race dyads. When different-race dyads were removed from the analysis, collaboration type differences disappeared. This result would suggest that social collaboration may be less helpful for different-race dyads than for same-race dyads. Similar to Experiment 1, participants reported positive interactions with their partner in the social dyad task. Most participants worked well together, reported that the task was completed as a team equally, and felt comfortable telling their partner when they disagreed. This suggests that the virtual social interaction resulted in potentially fruitful group dynamics.

\section{General Discussion}

We examined whether collaborative decision-making could improve accuracy for own- and other-race face identification. The effectiveness of both social and non-social collaboration, as well as racially homogeneous and diverse dyads, demonstrates the robust benefit of wisdom-of-crowds for improving own- and other-race face identification accuracy. There are theoretical and practical implications in the beneficial effects of collaborative decision-making in understanding how we recognize other-race faces. These implications fall into two categories: the generalizability across social and non-social collaboration and the generalizability across race. We consider each in turn.

Despite utilizing distinctive mechanisms, both social and non-social collaboration increase face identification accuracy, indicating that these diverse approaches benefit own- and other-race face recognition. Social collaboration takes advantage of the social feedback between the individuals [18], and is driven by the more accurate member of the dyad [17, 18]. In contrast, in non-social collaboration individual item-level responses are averaged, combining input equally from both members of the dyad [19, 20, 22]. Social and non-social collaboration improved performance for same-race dyads equally (Exp 1.), consistent with Jeckeln et al. (2018). Also consistent with this earlier result, the slight advantage for non-social collaboration found in Exp. 2 disappeared when only same-race dyads were retained in the analysis. Although we can speculate about the role of dyad diversity in the advantage for non-social collaboration, the lack of a significant interaction between dyad-composition and collaboration type, does not support a strong interpretation. We can conclude, however, that in the conditions we tested, social collaboration never proved more effective than non-social collaboration.

The consistently strong benefit of non-social collaboration may stem from its ability to avoid the possible negative outcomes of diversity in group decision making such as conflict [24, 29]. In this sense, non-social collaboration can exploit the strengths of different individuals, and thereby increase accuracy, while avoiding outcomes such as conflict, personality differences, and social awkwardness that may arise in the context of social collaboration. Of note, the overall improvement gains across collaboration type did not lessen the ORE, which suggests that the mechanisms by which collaboration improves identification of same-race faces can also be applied to other-race faces. Therefore, in practical terms it is rather unfeasible to expect collaboration to improve individual accuracy to a level equivalent to own-race recognition.

The comparable performance of same- and different- race dyads indicates that racial diversity, in this context, does not benefit general or race-specific face identification. It is plausible that the potential for race to benefit collaboration is complicated by the trade-off between diversity and ability [24, 25, 26], creating ambiguity as to which of these two should be prioritized for decision-making tasks. Given the strong and consistent effects of collaboration across the dyad conditions, it follows that different and effective strategies at the level of the individual participant (and not at the group level) is primarily responsible for collaborative benefits. As such, we demonstrate that wisdom of a multiracial crowd is not necessarily more advantageous than the wisdom of a homogeneous racial crowd.

As a tool for improving face identification accuracy, collaboration has multiple advantages over previous approaches. First, it is a face-image independent strategy that can be applied to any image. Second, in most cases, collaboration is more feasible and practical than learning from individuation training (e.g., [37]), multiple images (e.g., [14]), or caricatured faces (e.g., [12]). Third, here we demonstrate the versatility of collaboration benefits across racial groups. 
These strengths can be particularly useful for applied settings. For example, forensic facial examiners often collaborate on face identification decisions [20, 22]. If collaboration benefits can generalize to forensic practice settings for different race groups, this would prove a valuable avenue to assessing and improving the accuracy of forensic face identification decisions for other-race faces. Extending beyond this, fusion benefits have the potential to improve accuracy for other-race faces in the context of human-machine fusion [23, 22] and machine-machine fusion [22].

The shift to virtual collaboration to comply with social-distancing, may have impacted students interactions. It is possible that the online nature of the task affected the presence or magnitude of collaboration benefits. Therefore, it would be advisable to replicate these findings in an in-person setting. Also, the study was limited to two racial groups to enable the selection of images with controlled difficulty measures. Although there is no reason to believe the results would not generalize to other racial groups, this would need to be demonstrated empirically. Our findings suggest that it may be of interest to explore collaboration benefits across different contexts, including across age, gender, and image property variations. Also, the comparable results across dyad composition suggest that individuals within racial groups may have been using different, but equally efficient, strategies for identifying faces. Future work may consider how these individual approaches differ.

In summary, these experiments revealed the robust effects of collaboration across diverse groups, working together socially, or through combinations of their judgments, in recognizing faces of their own-race and of other races. Our findings have implications for the usefulness of collaborative decision-making in face identification tasks and address a gap in our knowledge about the scenarios in which collaboration can serve as a strategy for improving face identification accuracy.

\section{References}

[1] Roy S Malpass and Jerome Kravitz. Recognition for faces of own and other race. Journal of personality and social psychology, 13(4):330, 1969.

[2] Christian A Meissner and John C Brigham. Thirty years of investigating the own-race bias in memory for faces: A meta-analytic review. Psychology, Public Policy, and Law, 7(1):3, 2001.

[3] Innocence Project. Dna exonerations in the United States, 2020.

[4] Paul J Lavrakas, John R Buri, and Mark S Mayzner. A perspective on the recognition of other-race faces. Perception \& Psychophysics, 20(6):475-481, 1976.

[5] James W Tanaka and Lara J Pierce. The neural plasticity of other-race face recognition. Cognitive, Affective, \& Behavioral Neuroscience, 9(1):122-131, 2009.

[6] Sophie Lebrecht, Lara J Pierce, Michael J Tarr, and James W Tanaka. Perceptual other-race training reduces implicit racial bias. PloS one, 4(1):e4215, 2009.

[7] Joseph DeGutis, Christopher DeNicola, Tyler Zink, Regina McGlinchey, and William Milberg. Training with own-race faces can improve processing of other-race faces: Evidence from developmental prosopagnosia. Neuropsychologia, 49(9):2505-2513, 2011.

[8] Rankin W McGugin, James W Tanaka, Sophie Lebrecht, Michael J Tarr, and Isabel Gauthier. Race-specific perceptual discrimination improvement following short individuation training with faces. Cognitive science, 35(2):330-347, 2011.

[9] Sandy Sangrigoli and Scania De Schonen. Recognition of own-race and other-race faces by three-month-old infants. Journal of Child Psychology and Psychiatry, 45(7):1219-1227, 2004.

[10] Michelle Heron-Delaney, Gizelle Anzures, Jane S Herbert, Paul C Quinn, Alan M Slater, James W Tanaka, Kang Lee, and Olivier Pascalis. Perceptual training prevents the emergence of the other race effect during infancy. PloS one, 6(5), 2011.

[11] Gizelle Anzures, Andrea Wheeler, Paul C Quinn, Olivier Pascalis, Alan M Slater, Michelle Heron-Delaney, James W Tanaka, and Kang Lee. Brief daily exposures to asian females reverses perceptual narrowing for asian faces in caucasian infants. Journal of experimental child psychology, 112(4):484-495, 2012.

[12] Jobany Rodríguez, Heather Bortfeld, and Ricardo Gutiérrez-Osuna. Reducing the other-race effect through caricatures. In 2008 8th IEEE International Conference on Automatic Face \& Gesture Recognition, pages 1-5. IEEE, 2008.

[13] Jessica L Marcon, Christian A Meissner, Michael Frueh, Kyle J Susa, and Otto H MacLin. Perceptual identification and the cross-race effect. Visual Cognition, 18(5):767-779, 2010. 
[14] Jacqueline G Cavazos, Eilidh Noyes, and Alice J O'Toole. Learning context and the other-race effect: Strategies for improving face recognition. Vision research, 157:169-183, 2019.

[15] James Surowiecki. The wisdom of crowds. Anchor, 2005.

[16] Vicki Bruce, Zoë Henderson, Craig Newman, and A Mike Burton. Matching identities of familiar and unfamiliar faces caught on cctv images. Journal of Experimental Psychology: Applied, 7(3):207, 2001.

[17] Andrew J Dowsett and A Mike Burton. Unfamiliar face matching: Pairs out-perform individuals and provide a route to training. British Journal of Psychology, 106(3):433-445, 2015.

[18] Géraldine Jeckeln, Carina A Hahn, Eilidh Noyes, Jacqueline G Cavazos, and Alice J O'Toole. Wisdom of the social versus non-social crowd in face identification. British Journal of Psychology, 109(4):724-735, 2018.

[19] David White, A Mike Burton, Richard I Kemp, and Rob Jenkins. Crowd effects in unfamiliar face matching. Applied Cognitive Psychology, 27(6):769-777, 2013.

[20] David White, P Jonathon Phillips, Carina A Hahn, Matthew Hill, and Alice J O'Toole. Perceptual expertise in forensic facial image comparison. Proceedings of the Royal Society B: Biological Sciences, 282(1814):20151292, 2015.

[21] Bahador Bahrami, Karsten Olsen, Peter E Latham, Andreas Roepstorff, Geraint Rees, and Chris D Frith. Optimally interacting minds. Science, 329(5995):1081-1085, 2010.

[22] P Jonathon Phillips, Amy N Yates, Ying Hu, Carina A Hahn, Eilidh Noyes, Kelsey Jackson, Jacqueline G Cavazos, Géraldine Jeckeln, Rajeev Ranjan, Swami Sankaranarayanan, et al. Face recognition accuracy of forensic examiners, superrecognizers, and face recognition algorithms. Proceedings of the National Academy of Sciences, 115(24):6171-6176, 2018.

[23] Alice J O'Toole, Hervé Abdi, Fang Jiang, and P Jonathon Phillips. Fusing face-verification algorithms and humans. IEEE Transactions on Systems, Man, and Cybernetics, Part B (Cybernetics), 37(5):1149-1155, 2007.

[24] Richard L Moreland, JM Levine, and ML Wingert. Creating the ideal group: Composition effects at work. Understanding group behavior, 2:11-35, 2013.

[25] Shenghua Luan, Konstantinos V Katsikopoulos, and Torsten Reimer. When does diversity trump ability (and vice versa) in group decision making? a simulation study. PloS one, 7(2):e31043, 2012.

[26] Lu Hong and Scott E Page. Groups of diverse problem solvers can outperform groups of high-ability problem solvers. Proceedings of the National Academy of Sciences, 101(46):16385-16389, 2004.

[27] Samuel R Sommers. On racial diversity and group decision making: identifying multiple effects of racial composition on jury deliberations. Journal of personality and social psychology, 90(4):597, 2006.

[28] Poppy Lauretta McLeod, Sharon Alisa Lobel, and Taylor H Cox Jr. Ethnic diversity and creativity in small groups. Small group research, 27(2):248-264, 1996.

[29] Carsten KW De Dreu and Laurie R Weingart. Task versus relationship conflict, team performance, and team member satisfaction: a meta-analysis. Journal of applied Psychology, 88(4):741, 2003.

[30] J Westfall. Pangea: Power analysis for general ANOVA designs. 2015, 2015.

[31] Qualtrics. Qualtrics,2019, 2019.

[32] P Jonathon Phillips, J Ross Beveridge, Bruce A Draper, Geof Givens, Alice J O'Toole, David Bolme, Joseph Dunlop, Yui Man Lui, Hassan Sahibzada, and Samuel Weimer. The Good, the Bad, and the Ugly Face Challenge Problem. Image and Vision Computing, 30(3):177-185, 2012.

[33] Rajeev Ranjan, Ankan Bansal, Jingxiao Zheng, Hongyu Xu, Joshua Gleason, Boyu Lu, Anirudh Nanduri, JunCheng Chen, Carlos D Castillo, and Rama Chellappa. A fast and accurate system for face detection, identification, and verification. IEEE Transactions on Biometrics, Behavior, and Identity Science, 1(2):82-96, 2019.

[34] Katherine Y Williams and AO Charles. 'reilly. 1998. demography and diversity in organizations: A review of 40 years of research. Research in organizational behavior, 20(20):77-140, 1998.

[35] Elizabeth Mannix and Margaret A Neale. What differences make a difference? The promise and reality of diverse teams in organizations. Psychological science in the public interest, 6(2):31-55, 2005.

[36] John F Cross, Jane Cross, and James Daly. Sex, race, age, and beauty as factors in recognition of faces. Perception \& psychophysics, 10(6):393-396, 1971.

[37] James W Tanaka, Markus Kiefer, and Cindy M Bukach. A holistic account of the own-race effect in face recognition: Evidence from a cross-cultural study. Cognition, 93(1):B1-B9, 2004. 
[38] Cindy M Bukach, Jasmine Cottle, JoAnna Ubiwa, and Jessica Miller. Individuation experience predicts other-race effects in holistic processing for both caucasian and black participants. Cognition, 123(2):319-324, 2012.

[39] John C Brigham and Roy S Malpass. The role of experience and contact in the recognition of faces of own-and other-race persons. Journal of social issues, 41(3):139-155, 1985.

[40] Wei-Jen Ng and Rod CL Lindsay. Cross-race facial recognition: Failure of the contact hypothesis. Journal of Cross-Cultural Psychology, 25(2):217-232, 1994.

[41] Gillian Rhodes, Louise Ewing, William G Hayward, Daphne Maurer, Catherine J Mondloch, and James W Tanaka. Contact and other-race effects in configural and component processing of faces. British Journal of Psychology, 100(4):717-728, 2009.

[42] Pamela M Walker and Miles Hewstone. A perceptual discrimination investigation of the own-race effect and intergroup experience. Applied Cognitive Psychology: The Official Journal of the Society for Applied Research in Memory and Cognition, 20(4):461-475, 2006.

[43] Elinor McKone, Lulu Wan, Madeleine Pidcock, Kate Crookes, Katherine Reynolds, Amy Dawel, Evan Kidd, and Chiara Fiorentini. A critical period for faces: Other-race face recognition is improved by childhood but not adult social contact. Scientific reports, 9(1):1-13, 2019.

\section{Supplemental Section}

This supplemental material contains results, and discussion sections on the individuated experience survey administered in Experiment 2.

\subsection{Individuating other-race experience survey}

Correlations were computed in order to explore the relationship between individual and dyad face identification accuracy and self-reported qualitative and quantitative experience scores.

Correlations based on individual performance. We examined the relationship between other-race experience (quantitative and qualitative) individual other-race face identification accuracy. For East Asian participants, individual other-race identification accuracy was not correlated with self-reported individual other-race $(M=3.2, S D=.87)$ qualitative experience $(r(71)=-.14, p=.247)$, but it was negatively correlated with self-reported quantitative experience $(M=3.0, S D=1.0)(r(71)=-.24, p=.04)$. The latter result indicates that East Asians, with reportedly less quantitative experience with Caucasian individuals, performed more accurately than those with more experience, a seemingly counterintuitive finding. For Caucasian participants, individual other-race identification accuracy was not correlated with individual other-race qualitative experience $(M=2.8, S D=.90)(r(71)=0.03, p=.76)$, nor with quantitative experience $(M=1.9, S D=.70)(r(71)=.09, p=.418)$.

Correlations based on dyad performance. For dyad performance, we averaged individual other-race experience scores for each member of the dyad to create a composite dyad other-race experience score. Self-reported other-race qualitative and quantitative experience were not correlated with overall dyad performance for any dyad composition. For both same- and different-race dyads, qualitative other-race experience was not associated with other-race performance (East Asian dyads, $r(46)=-.004, p=.98$; East Asian/Caucasian dyads, $r(48)=.07, p=.64$; Caucasian dyads, $r(54)$ $=.12, p=.37)$. In contrast, quantitative other-race experience $(M=1.9, S D=.71)$ was positively correlated with other-race performance for Caucasian dyads only $(r(54)=.28, p=.03)$ (East Asian dyads, $r(46)=-.17, p=.25$; East Asian/Caucasian dyads, $r(48)=-.14, p=.33$ ).

\subsection{Discussion}

The relationship between other-race experience and other-race face identification accuracy remains ambiguous. Previous research found that experience can be either associated positively [41, 42, 37], negatively [4], or be negligibly to the magnitude of the ORE [1, 39, 40]. The distinction between of qualitative experience versus quantitative experience [4, 38] further complicates this relationship. Here, we add to this complex literature and provide some mixed findings in the context of same- and different-race dyads. For example, the negative relationship between other-race quantitative experience for East Asian participants and overall other-race identification accuracy may seem contradictory. One possibility is that a reported increase in quantitative exposure to other-race individuals inflated confidence in East Asian participants' ability to recognize other-race faces (i.e., they believed they were better equipped to identify Caucasian faces than they actually were). However, this negative relationship was not found consistently in other groups. In the context of dyad accuracy, Caucasian dyads showed an positive relationship between other-race quantitative experience 
and other-race face identification accuracy, which replicates some of the previous literature on other-race experience [38,4].

Taken together, these results provide a complex, tentative picture of the role of other-race experience in collaborative decision making. These mixed results, however, are unsurprising given the ambiguous findings in the literature about the role of experience in other-race face identification. Moreover, given the constraints of the COVID-19 pandemic, these findings should be considered with caution. Several components of the self-reported individuated experience surveys were designed to measure social interaction (e.g., "I often go to the houses of [East Asian/Caucasian] people", "I share meals with an [East Asian/Caucasian] individual at the same table while eating on campus."). The survey was not designed to capture experiences with other-race individuals in the context of a pandemic. Also, as previous research suggests, it is entirely possible that self-report experience with other-race individuals is not related to other-race identification performance [1, 40, 43]. An alternative hypothesis to explain the role of experience in the ORE is the developmental contact hypothesis [43]. This hypothesis would predict that only early developmental exposure to other-race faces can provide a protective barrier against the ORE. Most recently, the effect of developmental exposure to other-race individuals was demonstrated to be stronger indicator of the ORE than self-reported contact [43]. Notably, this type of experience is difficult to control, and was not captured in the present study. 